\title{
On the option pricing by the binomial model
}

\author{
Nikolaos Halidias \\ Department of Statistics and Actuarial-Financial Mathematics \\ University of the Aegean \\ Karlovassi 83200 Samos, Greece \\ email: nikoshalidias@hotmail.com
}

\begin{abstract}
In this note we study the binomial model applied to European, American and Bermudan type of derivatives. Our aim is to give the necessary and sufficient conditions under which we can define a fair value via replicating portfolios for any derivative using simple mathematical arguments and without using no arbitrage techniques. Giving suitable definitions we are able to define rigorously the fair value of any derivative without using concepts from probability theory or stochastic analysis therefore is suitable for students or young researchers. It will be clear in our analysis that if $e^{r \delta} \notin[d, u]$ then we can not define a fair value by any means for any derivative while if $d \leq e^{r \delta} \leq u$ we can. Therefore the definition of the fair value of a derivative is not so closely related with the absence of arbitrage. In the usual probabilistic point of view we assume that $d<e^{r \delta}<u$ in order to define the fair value but it is not clear what we can (or we can not) do in the cases where $e^{r \delta} \leq d$ or $e^{r \delta} \geq u$.
\end{abstract}

Keywords Option Pricing, Fair Value, Binomial Model, Bermudan Options

2020 Mathematics Subject Classification 91G20, 91G60

\section{Introduction}

In this note we study the option pricing problem via replicating portfolios using the binomial model in the spirit of [2] so one can refer to the literature for more details. Our aim is to define the fair value of any derivative in the binomial model setting without using probability theory and stochastic analysis. To do so we give suitable definitions and using replicating portfolios we are able to define the notion of the fair value. We see that this definition is not so closely related with the absence of arbitrage. In this spirit but with much more generality but also with much more mathematical complexity one can refer to [1] and the references therein.

We denote by $X$ any derivative on $[0, T]$, such as a call option or a lookback option etc. We will work on a discrete time setting, i.e. we divide $[0, T]$ into $N$ time intervals of length $\delta>0$ so that $N \delta=T$. We suppose that the holder of the derivative can exercise it at the times $e_{0}<e_{1}<\cdots<e_{m}=T$ where $e_{0}>0$, that is the holder has not the right to exercise the option at time zero. We denote by $0=k_{0}<k_{1}<\cdots<k_{l}<T$ all the other times in which the holder has not the right to exercise the option. We also denote by $S_{n}$ the value of the underlying stock of the derivative and by $X_{n}$ the payoff of the derivative at the time $n$ which in general is a function of $S_{0}, \cdots, S_{n}$. Our goal in this note is to give necessary and sufficient conditions to define a fair value for this derivative via replicating portfolios under the binomial model setting using simple 
mathematical arguments (i.e. without using probabilistic and stochastic analysis arguments) and without using no arbitrage techniques. For the probabilistic point of view one can see [1], [3], [8], [9], [10], [11] and [13].

We recall that in the binomial model one assumes that if the price of the underlying asset is $S_{n}$ at time $n$ then at time $n+1$ will be $u S_{n}$ or $d S_{n}$ for some $u>1$ and $d<1$.

We assume that one can invest in a risk free asset with continuously compounded interest rate $r$. That means that if someone invest 1 Euro for time period $\delta$ then this amount of money will become $e^{r \delta}$ Euros.

One can construct portfolios investing at the underlying asset and at the risk free asset. For example, at time zero one can buy $a_{0}$ shares of the underlying asset and also invest $b_{0}$ Euros at the risk free asset. Then, the value of this portfolio, at time zero, is $V_{0}=a_{0} S_{0}+b_{0}$ and at the time $n$ is $V_{n}=a_{n} S_{n}+b_{n}$ so we can denote this portfolio by $\left(a_{n}, b_{n}\right)$.

\section{Main Results}

The writer of the option would like to sell the derivative $X$ in a suitable price $V$. What that means? If with that amount of money the writer can construct a portfolio such that at the times $e_{n}$ its value is greater or equal to the value of the derivative, that is $V_{e_{n}} \geq X_{e_{n}}$, no matter what the value of the underlying asset is, then the writer can always pay the holder of the option. That is the main idea behind the option pricing, i.e. how to construct portfolios that replicates the derivative $X$.

Definition 1 We say that a portfolio $\left(a_{k}, b_{k}\right)$ replicates the derivative $X$ if $V_{e_{n}} \geq X_{e_{n}}$, for $n=0, \cdots, m$. We say that a replicating portfolio is acceptable by the writer if the portfolio replicates the derivative without the writer need to add funds until the exercise time but allows consumption. Finally, we say that an acceptable replicating portfolio is a honest portfolio if the holder can always exercise in a way that the value of the derivative equals to the value of this portfolio with zero consumption until the exercise time, i.e. there exists some time $e_{n}$ such that $V_{e_{n}}=X_{e_{n}}$ and that $\sum_{k=1}^{e_{n}} c_{k}=0$ where $c_{k}$ is the amount of money that the writer can consume at time $k$.

Is there any acceptable by the writer portfolio for the derivative $X$ ? If the writer constructs a portfolio at time zero, i.e. $V_{0}=a_{0} S_{0}+b_{0}$ then at time 1 its possible values are $V_{1}^{u}=a u S_{0}+b e^{r \delta}$ and $V_{1}^{d}=a d S_{0}+b e^{r \delta}$. For given $A, B \in \mathbb{R}$ we can construct a suitable portfolio at time zero such that its possible values at time 1 are $V_{1}^{u}=A$ and $V_{1}^{d}=B$. To do so we can choose $a_{0}=\frac{A-B}{(u-d) S_{0}}$ and $b_{0}=\frac{B u-A d}{e^{r \delta}(u-d)}$ and thus $V_{0}=e^{-r \delta}(q A+(1-q) B)$ where $q=\frac{e^{r \delta}-d}{u-d}$.

It is well known that an acceptable by the writer portfolio with possible consumption $c_{n}$ is given by the following

$$
\begin{aligned}
& V_{N} \quad=\quad X_{N} \\
& \text { and for } \quad n=0, \cdots, N-1 \text { we set } \\
& V_{n} \quad=\left\{\begin{array}{l}
\max \left\{X_{e_{j}}, e^{-r \delta}\left(q V_{e_{j}+1}^{u}+(1-q) V_{e_{j}+1}^{d}\right)\right. \\
e^{-r \delta}\left(q V_{k_{i}+1}^{u}+(1-q) V_{k_{i}+1}^{d}\right),
\end{array}\right. \\
& a_{n} \quad=\quad \frac{V_{n+1}^{u}-V_{n+1}^{d}}{(u-d) S_{n}}, \\
& b_{n}=\frac{V_{n+1}^{d} u-V_{n+1}^{u} d}{(u-d) e^{r \delta}},
\end{aligned}
$$

when $n=e_{j}$, for some $j=0, \cdots, m-1$

when $n=k_{i}$ for some $i=0, \cdots, l$ 


$$
c_{n}= \begin{cases}\max \left\{X_{e_{n}}-e^{-r \delta}\left(q V_{e_{n}+1}^{u}+(1-q) V_{e_{n}+1}^{d}\right), 0\right\}, & \text { when } n=e_{j}, \text { for some } j=0, \cdots, m-1 \\ 0, & \text { when } n=k_{i} \text { for some } i=0, \cdots, l\end{cases}
$$

Note that the above are related by the following equation $V_{n}=a_{n} S_{n}+b_{n}+c_{n}$ for $n=0, \cdots, N-1$ while $V_{N}=X_{N}$. If $V_{e_{n}}=X_{e_{n}}>e^{-r \delta}\left(q V_{e_{n}+1}^{u}+(1-q) V_{e_{n}+1}^{d}\right)$ for some $n$ and the holder do not exercise at that time then the writer can consume the amount $X_{e_{n}}-e^{-r \delta}\left(q V_{e_{n}+1}^{u}+(1-q) V_{e_{n}+1}^{d}\right)$. The value $V_{n}$ for $n=0, \cdots, N$ of the above portfolio is after the consumption of the amount $\sum_{k=1}^{n-1} c_{k}$. We will prove later that the above portfolio is always honest.

Definition 2 We call the above replicating portfolio fundamental and we denote it by $\left(a^{X}, b^{X}\right)$.

Our aim is to define a fair value for the option $X$. One candidate is the initial value of the fundamental portfolio. But then one can ask if there is another acceptable portfolio with less initial value so one can define this as a fair value. We will next study this question and we will see that if $e^{r \delta} \in[d, u]$ then there is not such a portfolio so the initial value of the fundamental portfolio is in fact the minimum one therefore we define the fair value as the initial value of $\left(a^{X}, b^{X}\right)$. Moreover, if $e^{r \delta} \notin[d, u]$ we will see that we can not define a fair value of the derivative by any means.

Theorem 3 The amount $V=e^{-r \delta}\left(q V_{n+1}^{u}+(1-q) V_{n+1}^{d}\right)$ equals to the minimum value of the portfolio at time $n$ such that the possible values of the portfolio at time $n+1$ are at least $V_{n+1}^{u}$ and $V_{n+1}^{d}$ if and if $d \leq e^{r \delta} \leq u$.

Proof.

- Suppose that $d \leq e^{r \delta} \leq u$ and suppose that there exists some $\varepsilon>0$ and $\varepsilon_{1}, \varepsilon_{2} \geq 0$ such that with the amount $V-\varepsilon$ we can find suitable $a^{\prime}, b^{\prime}$ with $V-\varepsilon=a^{\prime} S_{n}+b^{\prime}$ and $a^{\prime} u S_{n}+b^{\prime} e^{r \delta}=V_{n+1}^{u}+\varepsilon_{1}$ and also $a^{\prime} d S_{n}+b^{\prime} e^{r \delta}=V_{n+1}^{d}+\varepsilon_{2}$. Note that $V=a S_{n}+b$ where $a=\frac{V_{n+1}^{u}-V_{n+1}^{d}}{(u-d) S_{n}}$ and $b=\frac{V_{n+1}^{d} u-V_{n+1}^{u} d}{(u-d) e^{r \delta}}$.

Then it follows that

$$
\begin{aligned}
& a^{\prime}=a+\underbrace{\frac{\varepsilon_{1}-\varepsilon_{2}}{(u-d) S_{n}}}_{V_{1}} \\
& b^{\prime}=b+\underbrace{\frac{\varepsilon_{2} u-\varepsilon_{1} d}{(u-d) e^{r \delta}}}_{V_{2}}
\end{aligned}
$$

But

$$
V-\varepsilon=a^{\prime} S_{n}+b^{\prime}=a S_{n}+b+V_{1} S_{n}+V_{2}=V+V_{1} S_{n}+V_{2}
$$

Substituting for $V_{1}$ and $V_{2}$ it follows that

$$
\frac{\varepsilon_{1}\left(e^{r \delta}-d\right)+\varepsilon_{2}\left(u-e^{r \delta}\right)}{(u-d) e^{r \delta}}=-\varepsilon
$$

But this can be true only if $e^{r \delta} \notin[d, u]$ and that is a contradiction.

- Suppose that the amount $V=e^{-r \delta}\left(q V_{n+1}^{u}+(1-q) V_{n+1}^{d}\right)$ is the minimum amount of money as we describe above. We will prove that the inequality $d \leq e^{r \delta} \leq u$ must hold. Suppose that $e^{r \delta}<d$. Then for every $\varepsilon>0$ we choose $\varepsilon_{2}=0$ and $\varepsilon_{1}=\varepsilon \frac{(u-d) e^{r \delta}}{d-e^{r \delta}}$ and therefore the $a^{\prime}, b^{\prime}$ constructed as above defines a portfolio with at least the desired values at time $n+1$ and therefore we have a contradiction. The same holds for the case $u<e^{r \delta}$. 
Remark 4 In the case where the inequality $d \leq e^{r \delta} \leq u$ does not hold then with any amount $V_{n}$ at time $n$ (even negative) we can construct a suitable portfolio with at least the desired values at time $n+1$.

Definition 5 We denote by $R^{X}=\left\{V_{0}^{\left(a_{k}, b_{k}\right)}\right\}$ where $\left(a_{k}, b_{k}\right)$ is any acceptable by the writer replicating portfolio for the derivative $X$.

Note that $R^{X}$ is nonempty because it contains $V_{0}^{\left(a^{X}, b^{X}\right)}$ where $\left(a^{X}, b^{X}\right)$ is the fundamental replicating portfolio.

Corrolary 6 It holds that $d \leq e^{r \delta} \leq u$ if and only if the set $R^{X}$ is bounded from below. In the case where $R^{X}$ is bounded from below then the minimum is the amount $V_{0}^{\left(a^{X}, b^{X}\right)}$.

Proof. Note that the holder of the option do not have the right to exercise at time zero so $V_{0}=e^{-r \delta}\left(q V_{1}^{u}+\right.$ $\left.(1-q) V_{1}^{d}\right)$.

- Suppose that $d \leq e^{r \delta} \leq u$. Then using Theorem 3 repeatedly backwards for $n=N-1, \cdots, 0$ it follows that $R^{X}$ is bounded from below and the minimum is the amount $V_{0}^{\left(a^{X}, b^{X}\right)}$.

- Suppose that $R^{X}$ is bounded from below. We will prove that the inequality $d \leq e^{r \delta} \leq u$ holds. If it is not then by Theorem 3 it follows that $R^{X}$ is not bounded from below and this is a contradiction.

In the case where $R^{X}$ is bounded from below then the inequality $d \leq e^{r \delta} \leq u$ hold thus the minimum of $R^{X}$ is the amount $V_{0}^{\left(a^{X}, b^{X}\right)}$.

Definition 7 (Fair Value of the Derivative $X$ ) If $d \leq e^{r \delta} \leq u$ then the amount $V_{0}^{\left(a^{X}, b^{X}\right)}$ is defined as the fair value of the derivative $X$.

Note that if $e^{r \delta} \notin[d, u]$ then we can not define a fair value for an option (by any means not only via replicating portfolios) because we can replicate the derivative with any initial amount of money, even negative. But we can define a fair value if $d \leq e^{r \delta} \leq u$, i.e. even in the cases $d=e^{r \delta}$ or $u=e^{r \delta}$ in which there exists arbitrage opportunities. In the usual probabilistic point of view we assume that $d<e^{r \delta}<u$ in order to define the fair value but it is not clear what we can (or we can not) do in the cases where $e^{r \delta} \leq d$ or $e^{r \delta} \geq u$. Therefore the definition of the fair value of an option is not so closely related with the absence of arbitrage opportunities.

Remark 8 If $d=e^{r \delta}$ with the amount $e^{-r \delta}\left(q V_{n+1}^{u}+(1-q) V_{n+1}^{d}\right)$, setting $\varepsilon=\varepsilon_{2}=0$, we can construct a portfolio at time $n$ with at least the desired values at time $n+1$ for every $\varepsilon_{1}$. Similarly in the case where $u=e^{r \delta}$. That is, in these two cases (and of course in the case where $e^{r \delta} \notin[d, u]$ ) with the amount of money $e^{-r \delta}\left(q V_{n+1}^{u}+(1-q) V_{n+1}^{d}\right)$ we can construct acceptable by the writer replicating portfolios that are not honest.

Theorem 9 The fundamental replicating portfolio is always honest. In the binomial model it holds $d<e^{r \delta}<u$ if and if $R^{X}$ is bounded from below (in this case the minimum is $V_{0}^{\left(a^{X}, b^{X}\right)}$ ) and moreover with this amount of money we can not construct an acceptable by the writer portfolio that is not honest.

Proof. Recall first that $V_{e_{k}} \geq X_{e_{k}}$ for all $k=0, \cdots, m$. We will prove that the fundamental replicating portfolio is honest proving that, in every possible path of the value of the asset, there exists some $j=0, \cdots, m$ (which depends on that path) such that $V_{e_{j}}=X_{e_{j}}$ and with $\sum_{i=0}^{e_{j}} c_{i}=0$. Denote by

$$
j=\min \left\{k \in\{0, \cdots, m\}: V_{e_{k}}=X_{e_{k}}\right\}
$$


By construction of the fundamental replicating portfolio we have that $V_{N}=X_{N}$ so $e_{j} \leq e_{m}=N$. For this $j$ we obviously have that $V_{e_{j}}=X_{e_{j}}$ and that $c_{n}=0$ for $n=0, \cdots, e_{j}$ because for $n=0, \cdots, e_{j}$ either the holder do not have the right to exercise or $V_{n}>X_{n}$ when it has the right. Thus, there always exist some $j \in\{0, \cdots, m\}$ such that $V_{e_{j}}=X_{e_{j}}$ and $\sum_{i=0}^{e_{j}} c_{i}=0$.

- Let $d<e^{r \delta}<u$. By Corollary 6 we have that $R^{X}$ is bounded from below and the minimum is $V_{0}^{\left(a^{X}, b^{X}\right)}$. Moreover by the proof of Theorem 3 it follows that $\varepsilon=\varepsilon_{1}=\varepsilon_{2}=0$ so with the amount $V_{0}^{\left(a^{X}, b^{X}\right)}$ we can not construct other acceptable by the writer portfolio that is not honest.

- Suppose that $R^{X}$ is bounded from below, in this case the minimum is $V_{0}^{\left(a^{X}, b^{X}\right)}$, and that with this amount of money we can not construct an acceptable by the writer portfolio that is not honest. By Corollary 6 we obtain the inequality $d \leq e^{r \delta} \leq u$. Suppose that $d=e^{r \delta}$ or $u=e^{r \delta}$. Then by Remark 8 it follows that with the amount $V_{0}^{\left(a^{X}, b^{X}\right)}$ we can construct an acceptable by the writer replicating portfolio that is not honest. So we have a contradiction, that is strict inequalities should hold.

The time $j$ as described in the proof of the previous theorem is called optimal exercise time in the literature. Note that this does not mean that if the holder exercise at that time he/she will make the best profit. For a discussion on this matter, i.e. the best possible profit of the holder, one can see [4].

Remark 10 If we suppose that there exists some $\sigma>0$ such that $u=e^{\sigma \sqrt{\delta}}$ and $d=e^{-\sigma \sqrt{\delta}}$ (which is not in general the case) then we can choose small enough $\delta$ so that the inequality $d<e^{r \delta}<u$ holds. In this case one can show that $V_{0}(\delta) \rightarrow V_{0}$ (see [14]) which is connected with the Black-Scholes equation. It is well known that the binomial model does not admit arbitrage opportunities if and only if $d<e^{r \delta}<u$. So, by Theorem 9, it follows that the binomial model does not admit arbitrage if and only if $R^{X}$ is bounded from below (in this case the minimum is $\left.V_{0}^{\left(a^{X}, b^{X}\right)}\right)$ and moreover with this amount of money we can not construct an acceptable by the writer portfolio that is not honest.

\section{Conclusion}

In the binomial model setting we have given necessary and sufficient conditions in order to define the fair value of any derivative via replicating portfolios. Our analysis uses simple mathematical tools but we have not use any no arbitrage arguments. It is clear that if $e^{r \delta} \notin[d, u]$ then one can not define a fair value by any means because one can construct a replicating portfolio with any initial value. Moreover, we can define a fair value even in the cases where $e^{r \delta}=d$ or $e^{r \delta}=u$ and that means that the definition of the fair value is not so closely related with the absence of arbitrage. Finally, we have related the absence of arbitrage opportunities in the binomial model with the definition of the fair value of any derivative and with the construction of honest acceptable portfolios.

\section{References}

[1] Burzoni, M. - Frittelli, M. - Hou, Z. - Maggis, M. - Obloj, J. Pointwise arbitrage pricing theory in discrete time. Math. Oper. Res. 43, 1034 - 1057 (2019).

[2] J. Cox - S. Ross - M. Runinstein, Option Pricing: A Simplified Approach, Journal of Financial Economics 7 (1979) 229-263.

[3] Durrell Duffie, Dynamic Asset Pricing Theory, Princeton University Press, 2001. 
[4] N. Halidias, An elementary approach to the option pricing problem, Asian Research Journal of Mathematics, 1 (1), 1-18, 2016.

[5] H. He, Convergence from the discrete to continuous time contingent claim prices, Review of Financial Studies, vol. 3, p. 523-546, 1990.

[6] J. Hull, Options, Futures and Other Derivatives, Prentice Hall, 2010.

[7] R. Korn - E. Korn, Option Pricing and Portfolio Optimization, AMS, 2000.

[8] I. Karatzas - S. Shreve, Methods of Mathematical Finance, Springer, 1998.

[9] M. Musiela - M. Rutkowski, Martingale Methods in Financial Modelling, Springer, 2005.

[10] A. Pascucci-W. Runggaldier, Financial Mathematics, Springer, 2012.

[11] S. Shreve, Stochastic Calculus for Finance I and II, Springer, 2004.

[12] P. Wilmott, Paul Wilmott on Quantitative Finance, Wiley, 2007.

[13] Lishang Jiang, Mathematical Modeling and Methods of Option Pricing, World Scientific, 2005.

[14] Lishang Jiang and Min Dai, Convergence of Binomial Tree Methods for European/American Pathdependent Options, SIAM Journal on Numerical Analysis, Vol. 42, No. 3, pp. 1094-1109, 2004. 